\section{Quand l'ostéoblaste courtise la cellule souche}

Laure Coulombel
Inserm U.421,

Faculté de Médecine,

8, rue du Général Sarrail,

94010 Créteil, France.

coulombel@im3.inserm.fr
> Selon Snoopy, les grandes idées ne nous viennent que sur le toit de notre niche... C'est aussi l'avis des cellules souches hématopoïétiques (CSH): sorties de leur contexte tissulaire in vivo, elles refusent souvent obstinément de se diviser et perdent leur potentiel à notre grand désespoir. L'alibi facile est d'attribuer cet échec à la difficulté qu'il y a à reconstituer in vitro la complexité de la «niche» tissulaire où évoluent ces cellules souches, intrication complexe, et probablement très fluctuante, de contacts cellulaires et de molécules sécrétées. C'est à Mike Dexter que revient le mérite d'avoir en 1977 établi in vitro une «niche hématopoïétique» constituée des cellules adhérentes (dites «stromales») issues du prélèvement de moelle osseuse [1]. Ces cellules associent myofibroblastes, cellules endothéliales, fibroblastes, leur matrice extracellulaire, et un halo de protéoglycanes et de glycosaminoglycanes auxquels s'adsorbent des cytokines et autres molécules régulatrices. Certains progéniteurs, proches par leur fonction des cellules souches (et appelés LTC-IC, long-term culture initiating cells), ont adopté ce «microenvironnement», dans lequel ils se «nichent», s'autorenouvellent et se différencient pendant plusieurs mois chez la souris, mais seulement 8 - 12 semaines chez l'homme, d'où le nom de cultures à long terme donné à ce système de culture [2]. Mais 20 ans après les travaux de $M$. Dexter, en dépit d'un nombre considérable de travaux, on ne connaît toujours pas précisément le mélange d'ingrédients qui fait de cet environnement le support indispensable à l'expression des propriétés des CSH. Des molécules comme Wnta, les ligands des récepteurs Notch, certains composants de la matrice extracellulaire, certaines cytokines transmembranaires (ligand de FLT-3, SCF [stem cell factor], FGF [fibroblast growth factor], thrombopoïétine), des molécules d'adhérence, ont été individuellement identifiées comme des régulateurs importants de ces LTC-IC ou des cellules souches identifiées in vivo, mais leur contribution respective, leur cinétique d'expression dans un réseau tridimensionnel extrêmement complexe et très fluctuant, sont inconnues. Certains, dont Pierre Charbord [3], se sont récemment attachés à définir le profil transcriptionnel de cellules stromales «compétentes» et à le comparer à celui de cellules stromales inefficaces, mais de même qu'aucun gène spécifique ne permet de définir une cellule souche, aucun gène spécifique - mais plutôt les modifications d'un groupe de gènes - ne permet de distinguer une bonne «niche» d'une mauvaise niche. Deux articles publiés récemment dans Nature [4, 5] attribuent aux cellules osseuses ostéoblastiques (rarement incluses dans les populations cultivées in vitro) un rôle primordial in vivo dans la régulation du nombre des CSH. Le grand intérêt de ces articles est que l'analyse du microenvironnement est faite in vivo, grâce à l'exploitation de modèles murins génétiques, et il s'y associe une étude histologique très détaillée des modifications identifiées. On sait depuis longtemps que la répartition des CSH ne se fait pas au hasard dans les os longs (voir
Figure 1): elles sont préférentiellement localisées au contact des lamelles osseuses en formation, issues des ostéoblastes, et qui après leur minéralisation formeront l'os compact [6] (Figure IB, C). Les précurseurs plus matures migrent vers les vaisseaux de la cavité médullaire centrale dans lesquels ils sont relargués en fin de maturation $[7,8]$. Les cellules souches dont il est question dans ces articles sont définies par leur phénotype, Lin-Scal ${ }^{+} \mathrm{c}-\mathrm{kit}^{+}$, par leur cycle cellulaire lent, comme l'atteste la rétention du BrdU, mais surtout par leur capacité de reconstituer à long terme l'hématopoï̀se d'une souris irradiée létalement. Le nombre de CSH peut être défini avec précision dans des expériences de reconstitution effectuées avec des nombres décroissants de cellules (dilution limite). Les deux équipes ont utilisé la même stratégie: induction d'une variation du nombre d'ostéoblastes et analyse du comportement des CSH. J. Zhang et al. [4] ont postulé que le signal transmis par les protéines BMP (bone morphogenic protein) était important, compte tenu de son rôle dans l'induction de l'hématopoïèse chez l'embryon; ils ont éteint de façon conditionnelle le gène codant pour le récepteur de bmpl (bmprla), par l'intermédiaire d'un mutant Creinductible (promoteur $\mathrm{mxl}$ inductible par Polyl:C) et testé le retentissement sur les ostéoblastes, la production osseuse et les CSH; L.M. Clavi et al. [5] ont, quant à eux, induit un accroissement de la population des ostéoblastes par la surexpression, ciblée dans les cellules osseuses, du gène codant pour les 


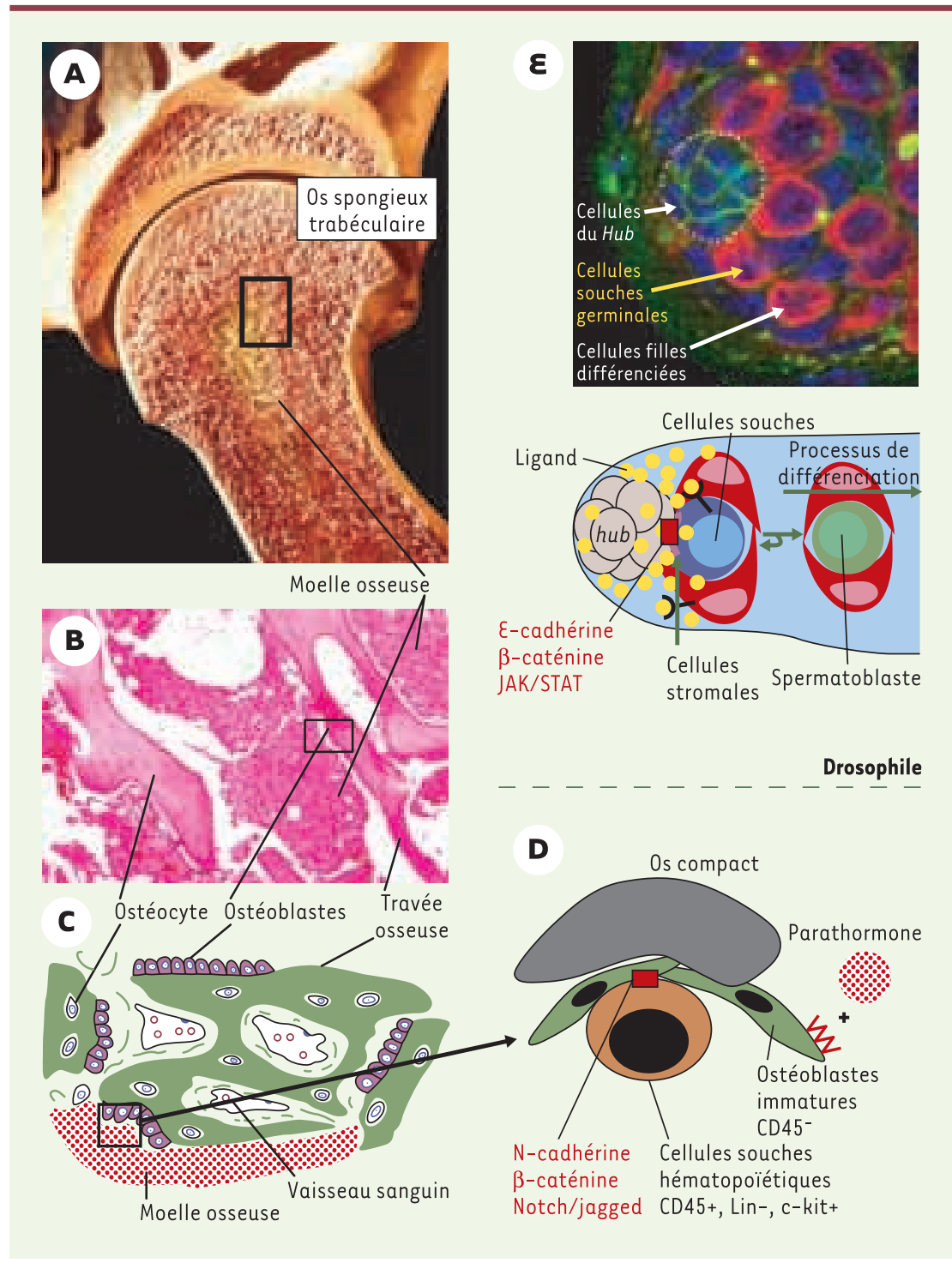

Figure 1. Organisation de la niche hématopoïétique. A. Les cellules souches hématopoïétiques résident dans l'os spongieux des os longs, au niveau des travées osseuses en formation, à la jonction épiphyse-métaphyse. B. Coupe histologique faite à partir d'une biopsie de moelle osseuse (os long, épine iliaque). Les multiples travées osseuses délimitent des logettes où se multiplient et se différencient les progéniteurs hématopoïétiques; ces logettes sont bordées sur leur face hématopoïétique par les ostéoblastes immatures (C), qui se transforment en ostéocytes présents dans l'os compact $(C, D)$; les travées délimitent les logettes hématopoiétiques, et dans ces logettes, les cellules souches sont localisées au contact des ostéoblastes, en périphérie de la logette. $D$. La jonction entre les cellules souches et les ostéoblastes fait intervenir la cadhérine $N$, la $\beta$-caténine, les récepteurs Notch-1 et 2 et leurs ligands (dont Jagged-1), et la densité de la population d'ostéoblastes est modulée par la parathormone. $\varepsilon$. Cette organisation rappelle celle que l'on rencontre dans la gonade mâle de la drosophile, où les cellules souches germinales, qui se différencieront en spermatoblastes sont au contact des cellules somatiques et des cellules du hub qui contrôlent leur autorenouvellement par le biais de molécules solubles et d'adhérence (nous remercions Erika Manutis [Carnegie Institute] pour ces documents sur la drosophile) [11]. récepteurs de la parathormone (PTH) et du peptide associé (PTHRp, PTH related peptide). L'administration de PTH à des souris normales reproduira les observations faites dans les souris surexprimant le récepteur PTHR. L'extinction de bmprla a entraîné la formation ectopique d'os trabéculaire, par un mécanisme encore mal compris, ce qui s'accompagne d'une augmentation inappropriée du nombre d'ostéoblastes. Dans les deux modèles, l'augmentation des ostéoblastes s'accompagnait d'une augmentation concomitante du nombre de cellules souches, identifiées dans les tests évoqués ci-dessus. Curieusement, I'inflation concernait le seul compartiment des CSH, mais épargnait celui des précurseurs hématopoïétiques plus matures, descendant des CSH. Une restriction semblable avait été notée dans les souris dont les CSH avaient été transduites avec le gène hoxb4, et suggère la conservation des rétrocontrôles négatifs. Le groupe de J. Zhang et al. a analysé de façon extrêmement précise la distribution anatomique des $\mathrm{CSH}$ et des ostéoblastes immatures (exprimant la $\mathrm{N}$-cadhérine, mais dépourvues de l'antigène CD45) dans les formations ectopiques de cloisons osseuses se développant en l'absence de signalisation Bmpla. C'est au contact de ces ostéoblastes, et seulement là, que s'accumulent les CSH (repérées sur coupes histologiques par leur rétention de BrdU, prouvant un cycle cellulaire lent). Encore plus intéressante, l'observation selon laquelle la $\mathrm{N}$-cadhérine est exprimée sur une sous-population de CSH, et la localisation de cette molécule en regard de celle de la $\beta$-caténine implique cette famille de molécules d'adhérence (Figure 1D). Le parallèle avec la drosophile est particulièrement frappant (Figure $1 \varepsilon$ ): dans ce modèle, ce sont également la $\varepsilon$-cadhérine et la $\beta$-caténine qui ancrent les cellules souches germinales (CSG) aux cellules somatiques au pôle de la gonade [9], et I'on sait que, dans ce fabuleux modèle, l'autorenouvellement des CSG est sous le 
contrôle de cellules somatiques, et fait intervenir l'adhérence entre les deux types cellulaires, des molécules solubles via la voie JAK-STAT, et une orientation du fuseau mitotique dictée par l'arrimage du centrosome dans ce point d'adhérence aux cellules somatiques (Figure l $1 \varepsilon$ ) $(\rightarrow)$. Mais dans l'os, le seul contact entre $(\rightarrow) \mathrm{m} / \mathrm{s}$ 2003, $n^{\circ} 12$, p. 1201 ostéoblaste et CSH ne suffit pas: un troisième larron est indispensable: l'activation d'un récepteur Notch (Notchl ou-2), exprimé par les CSH, par un de ses ligands, jagged 1, dont l'ex- pression est augmentée en réponse à l'activation des récepteurs PTHR. L'implication de cette voie de signalisation n'est pas étonnante, elle est considérée maintenant comme l'un des médiateurs essentiels du choix cornélien qu'une CSH doit faire entre autorenouvellement et «détermination (commitment)», et pas seulement dans le système hématopoïétique [10]. La drosophile, là encore, nous l'a depuis longtemps démontré sans ambiguïté. Ces études sont la confirmation moléculaire in vivo de l'importance des interactions entre les cellules osseuses et les CSH, suspectées par des études anatomiques anciennes de la répartition des CSH et des précurseurs dans la moelle osseuse, et plus récemment par l'observation selon laquelle l'ostéoblaste est une cellule présentatrice de cytokines pour les progéniteurs hématopoḯtiques. Dommage que Pierre Dac ne puisse nous commenter cette nouvelle histoire de L'os à moelle... $\diamond$

Intimate interactions

between osteoblasts

and hematopoietic stem cells

\section{RéFÉRENCES}

1. Dexter TM, Allen TD, Lajtha LG. Conditions controlling the proliferation of haemopoietic stem cells in vitro. J Cell Physiol 1977; 91: 335-44.

2. Coulombel L, Eaves $A C$, Eaves CJ. Enzymatic treatment of long-term human marrow cultures reveals the preferential location of primitive hemopoietic progenitors in the adherent layer. Blood 1983; 62: 291-7.

3. Hackney JA, Charbord P, Brunk BP, Stoeckert CJ, Lemischka IR, Moore KA. A molecular profile of a hematopoietic stem cell niche. Proc Natl Acad Sci USA 2002; 99: 13061-6.
4. Zhang J, Niu C, Ye L, et al. Identification of the haematopoietic stem cell niche and control of the niche size. Nature 2003; 425: 836-41.

5. Calvi LM, Adams GB, Weibrecht KW, et al. Osteoblastic cells regulate the haematopoietic stem cell niche. Nature 2003 ; 425: 841-6.

6. Ducy P. Contrôle génétique de la squelettogenèse. Med Sci (Paris) 2001; 17: 1242-51.

7. Nilsson SK, Johnston HM, Coverdale JA. Spatial localization of transplanted hemopoietic stem cells: inferences for the localization of stem cell niches. Blood 2001; 97: 2293-9.
8. Lord BI, Wright \&G. Spatial organisation of CFU-S proliferation regulators in the mouse femur. Leuk Res $1984 ; 8: 1073-83$.

9. Spradling A, DrummondBarbosa D, Kai T. Stem cells find their niche. Nature 2001; 414: 98-104.

10. Maillard I, Adler SH, Pear WS. Notch and the immune system. Immunity 2003. 19: 781-91.
11. Tulina N, Matunis $\varepsilon$. Control of stem cell self-renewal in Drosophila spermatogenesis by JAK-STAT signaling. Science 2001; 294: 2546-9. 